\title{
Revealing Failure Modes and Effect of Catalyst Layer Properties for PEM Fuel Cell Cold Start using an Agglomerate Model
}

\author{
Liu Yang ${ }^{1}$, Chenxi Cao ${ }^{1}$, Quanquan $\mathrm{Gan}^{2}$, Hao Pei ${ }^{1}$, Qi Zhang ${ }^{1}$, and Ping $\mathrm{Li}^{1}$ \\ ${ }^{1}$ East China University of Science and Technology \\ ${ }^{2}$ Tsinghua University
}

September 24, 2021

\begin{abstract}
We propose a dynamic proton exchange membrane fuel cell model for cold start simulation in account for reactant transport, water phase-transfer and electrochemical reactions within catalyst agglomerates. The competition between in-agglomerate concentration loss and coverage of active electrocatalytic surface is shown to create different failure modes for low and high start-up currents. The critical ice fraction of failure was studied for different catalyst layer (CL) thickness and ionomer to carbon ratios (I/C) at $0.4 \mathrm{~A} \mathrm{~cm}-2$. Thicker cathode CLs allow cold start to proceed with higher ice fractions. In contrast, larger cathode I/Cs causes cold-start failure at lower ice fractions because of reduction of the CL porosity and agglomerate pore sizes that significantly increases the oxygen transport resistance. By utilizing the electro-osmotic drag effect, slightly thick anode CL could act as effective heat sources during cold start at high currents with minimal impact on the nominal cell performance.
\end{abstract}

\section{Hosted file}

Manuscript_Two_Dimensional_PEMFC_Stack_Agglomerate_Model-aic4'.pdf available at https : //authorea.com/users/435155/articles/538367-revealing-failure-modes-and-effect-ofcatalyst-layer-properties-for-pem-fuel-cell-cold-start-using-an-agglomerate-model 
(a)

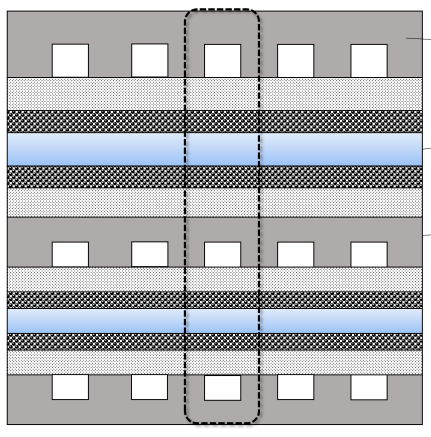

(c)

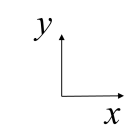

$\vec{x}$

C

(b)

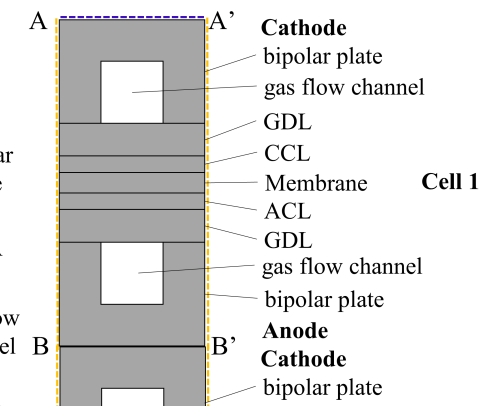

gas flow channel

GDL

Membrane Cell 2

ACL

GDL

- gas flow channel

bipolar plate

Anode

symmetric boundary condition ---.-convective boundary condition -------

Secondary

pore Oxygen

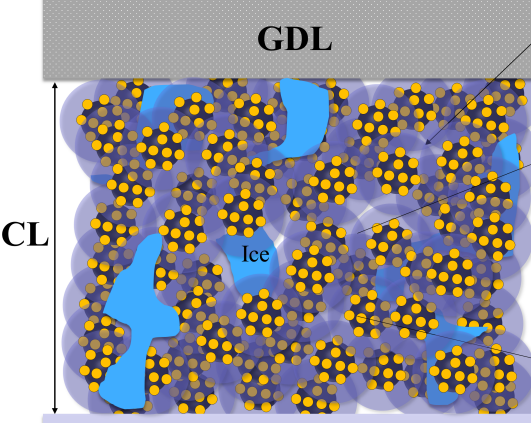

Membrane

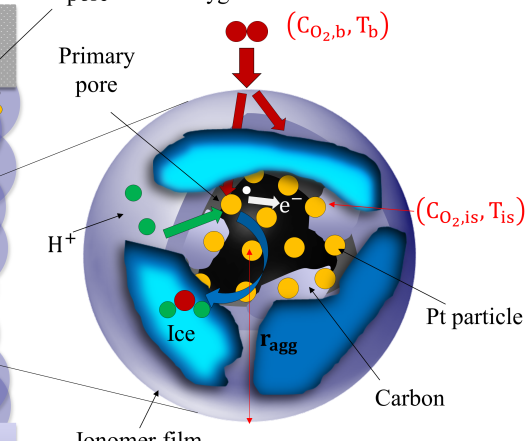

Ionomer film 
(a)

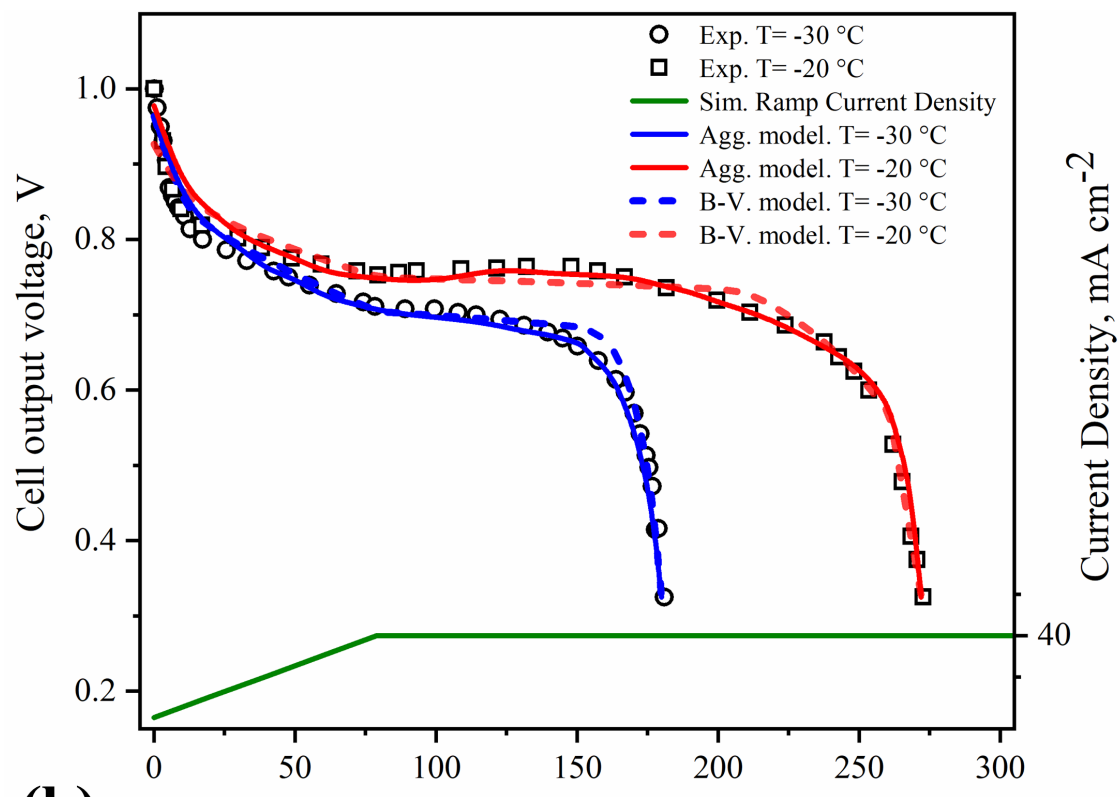

(b)

Evolution time, $\mathrm{s}$

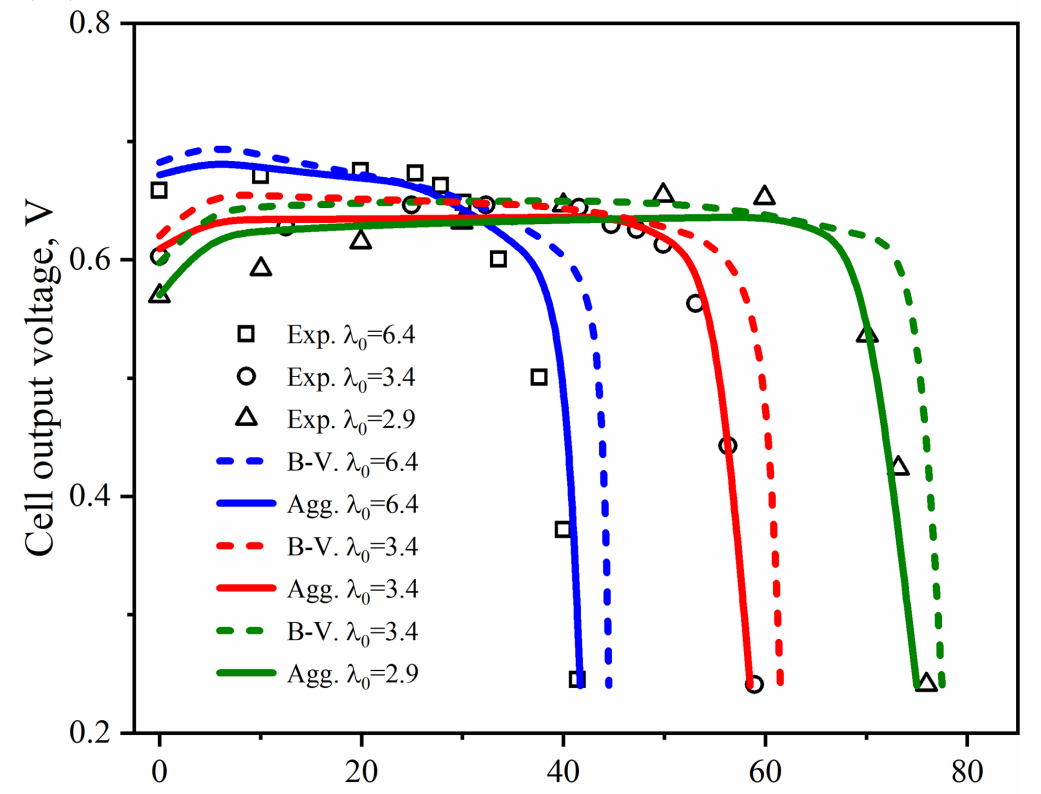

(c)

Evolution time, $\mathrm{s}$

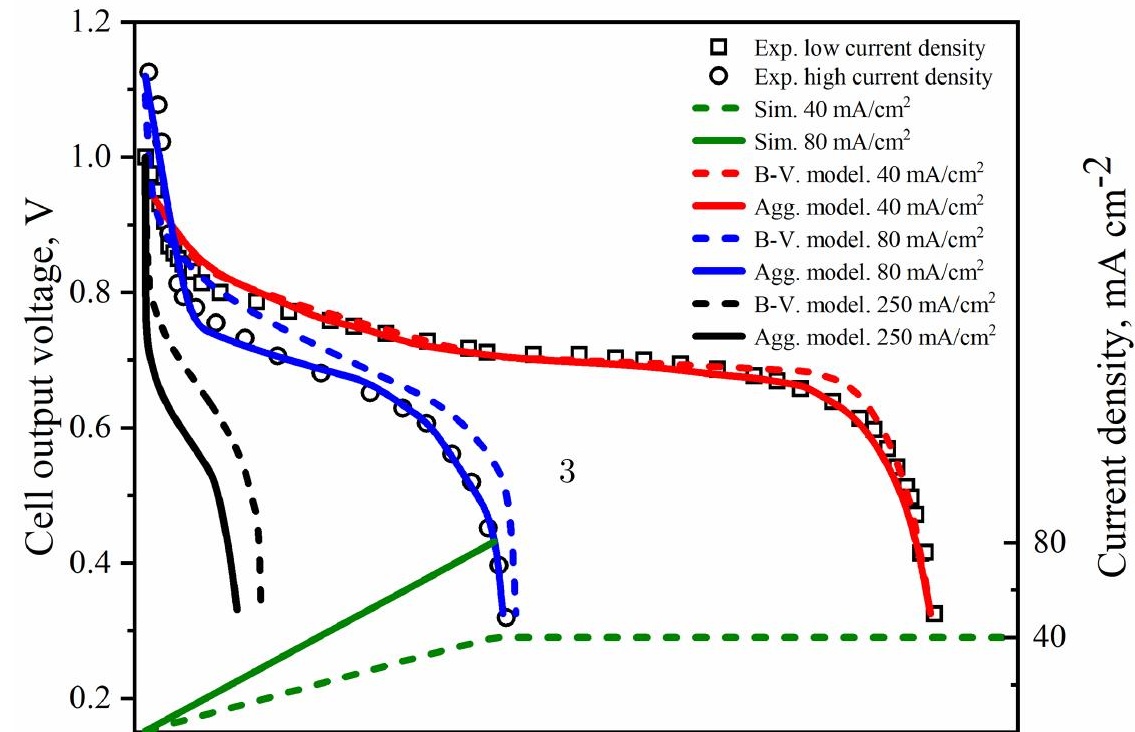



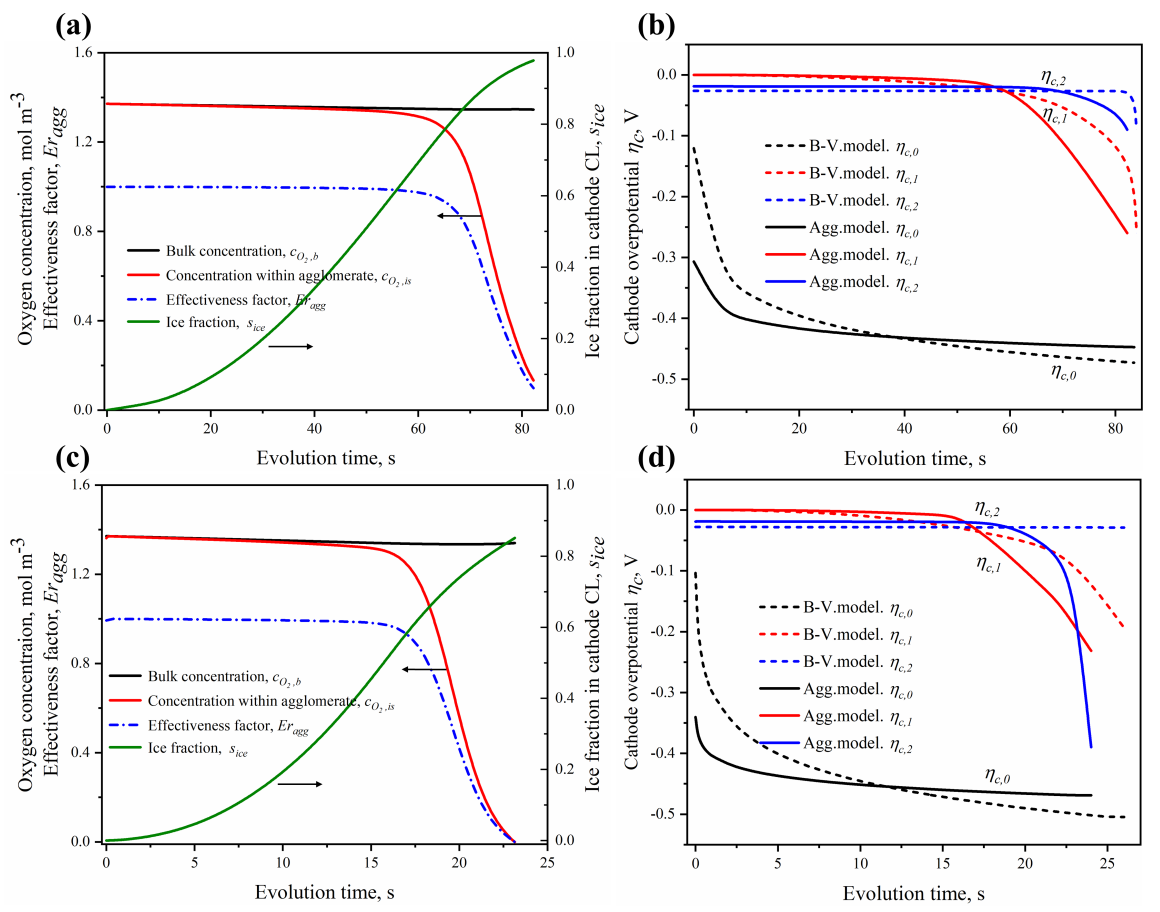

(a)
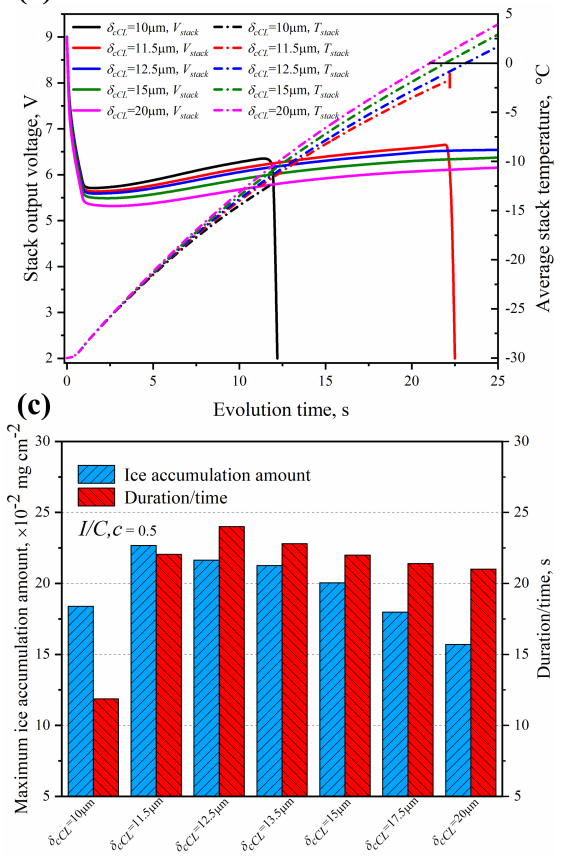

(b)
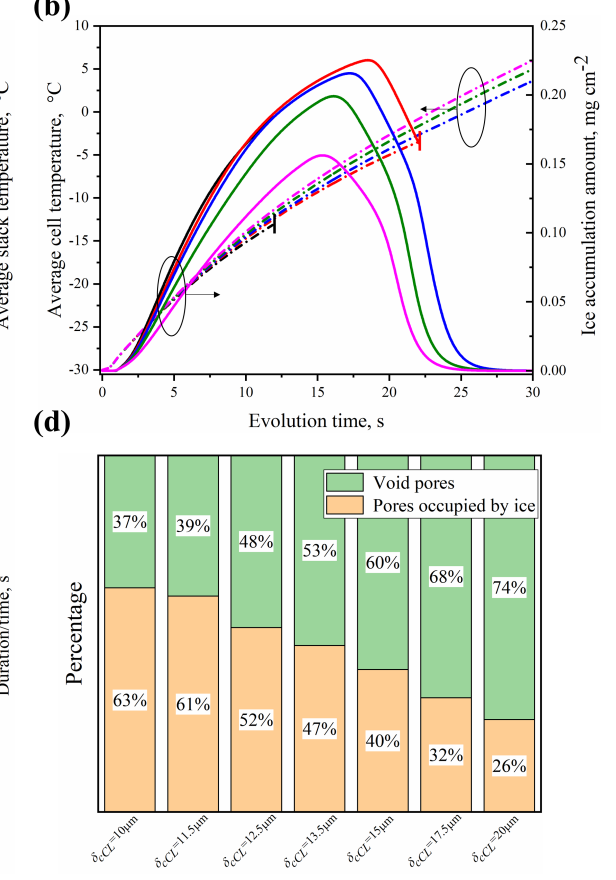

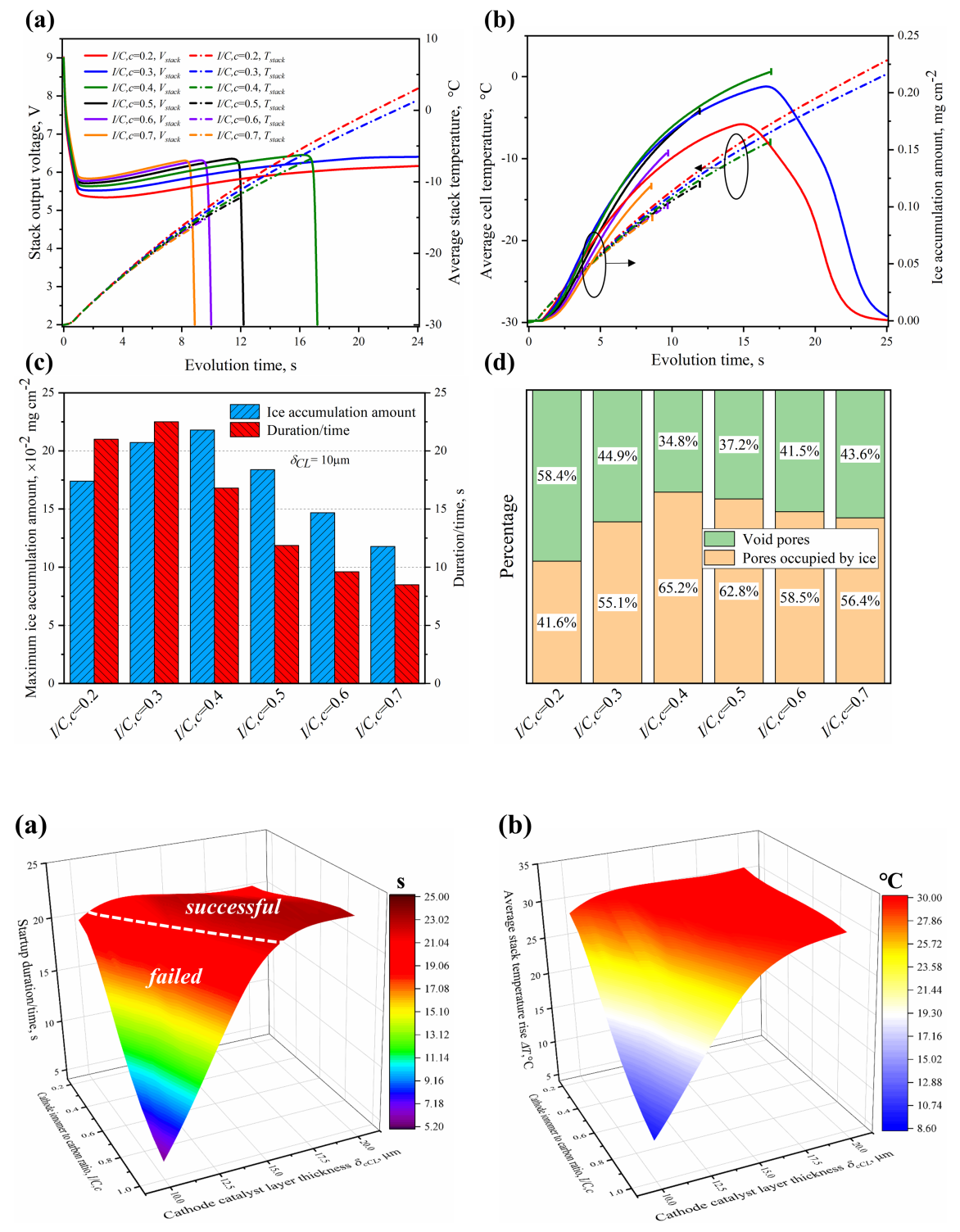
(a)

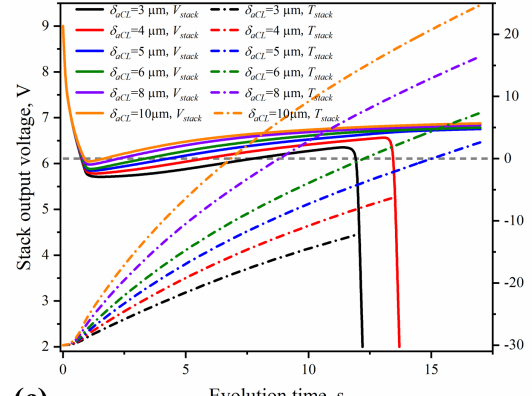

(c)

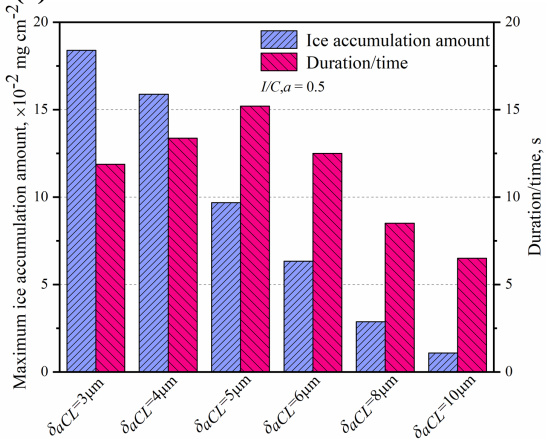

(b)

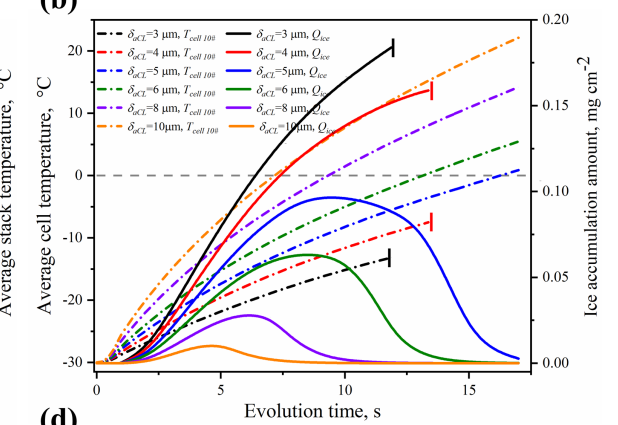

(d)

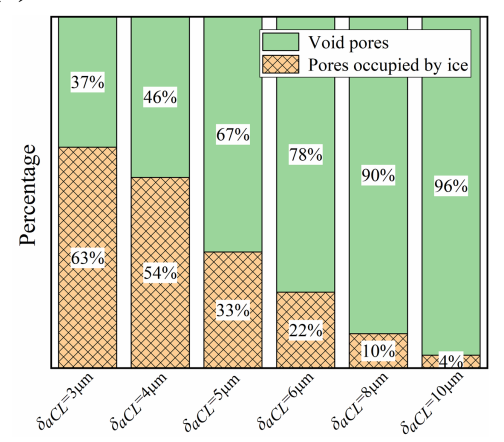

(a) Cell 1\#

Cell 10\# ${ }^{\circ} \mathrm{C}$

$\mathrm{t}=15 \mathrm{~s}$

$\mathrm{t}=12 \mathrm{~s} / \mathrm{W}$ |

$\mathbf{t = 9 s}$ :

$\mathrm{t}=3 \mathrm{~s}$ :

$\mathrm{t}=0.8 \mathrm{~s}$
anode cathode

(b) Cell 1\#

$\mathrm{t}=28 \mathrm{~s}$

$\mathrm{t}=\mathbf{2 0 s}$ : |

t=15s

$\mathrm{t}=5 \mathrm{~s}$ [

t=1s anode cathode 
(a) $\delta_{a C L}=6 \mu \mathrm{m} \quad \delta_{c C L}=10 \mu \mathrm{m}$

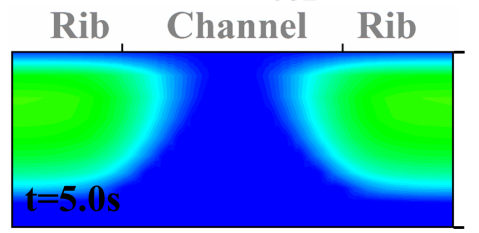

Rib Channel Rib

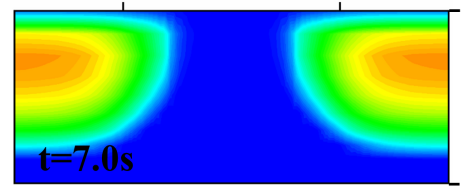

Rib Channel Rib

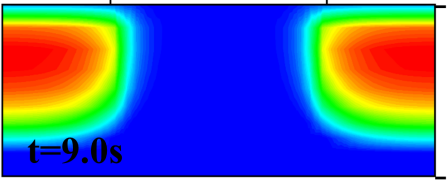

Rib Channel Rib

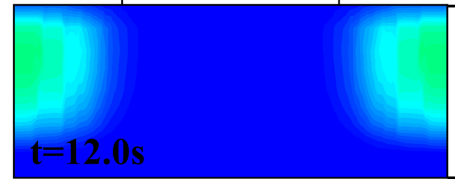

$\delta_{a C L}=3 \mu \mathrm{m} \quad \delta_{c C L}=20 \mu \mathrm{m}$
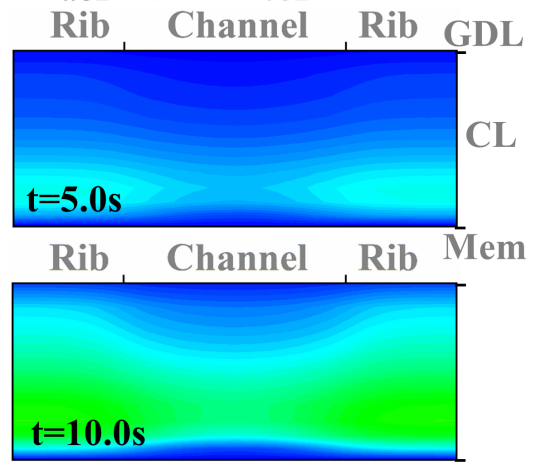

Rib, Channel, Rib
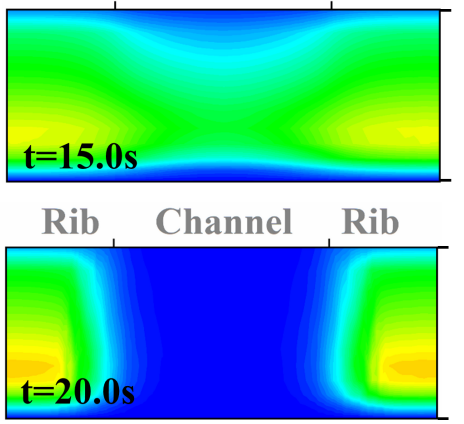

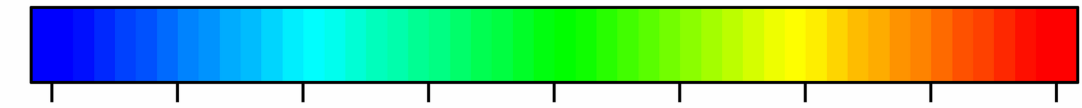

$\begin{array}{lllllllll}0.0000 & 0.0763 & 0.1525 & 0.2288 & 0.3050 & 0.3813 & 0.4575 & 0.5338 & 0.6100\end{array}$

(b) $\delta_{a C L}=6 \mu \mathrm{m} \quad \delta_{c C L}=10 \mu \mathrm{m}$

Rib Channel Rib

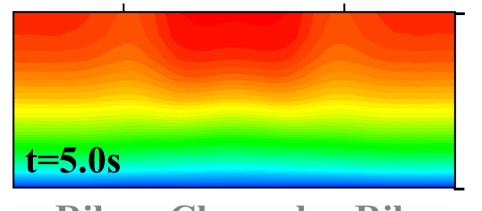

Rib Channel Rib

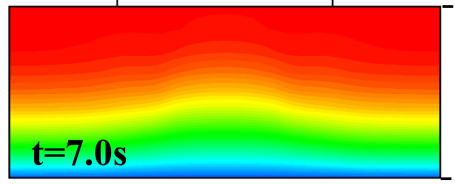

Rib Channel Rib

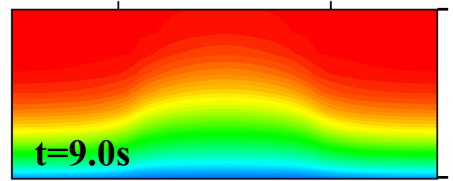

Rib Channel Rib

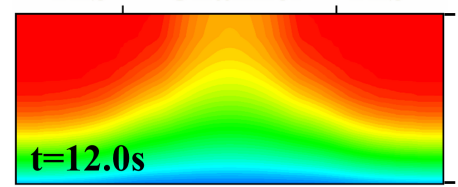

$\delta_{a C L}=3 \mu \mathrm{m} \quad \delta_{c C L}=20 \mu \mathrm{m}$
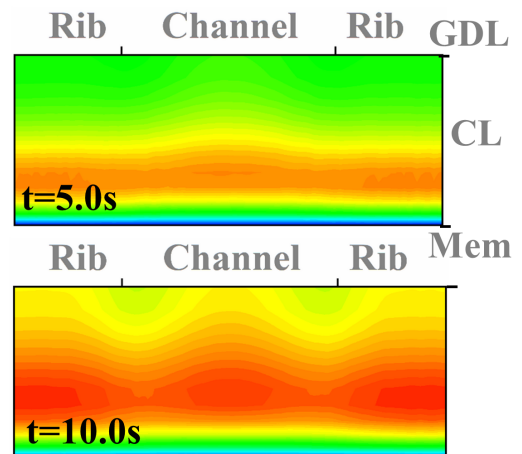

Rib Channel Rib

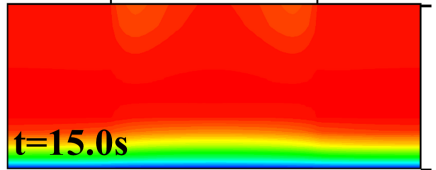

Rib Channel Rib

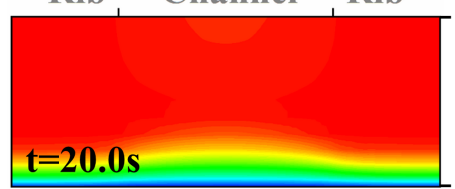

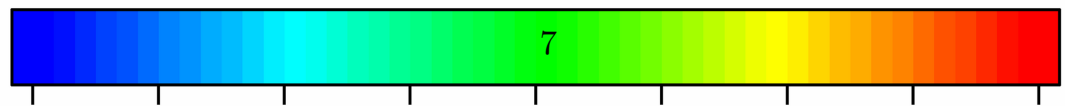
3.95
5.59 


\section{Hosted file}

Tables_Two_Dimensional_PEMFC_Stack_Agglomerate_Model-aic.docx available at https://authorea. com/users/435155/articles/538367-revealing-failure-modes-and-effect-of-catalyst-layerproperties-for-pem-fuel-cell-cold-start-using-an-agglomerate-model 